\title{
Effects of Electronic Nicotine Delivery Systems and Cigarettes on Systemic Circulation and Blood-Brain Barrier
}

\section{Implications for Cognitive Decline}

\author{
Nathan A. Heldt, ${ }^{\star \dagger}$ Nancy Reichenbach, ${ }^{*}$ Hannah M. McGary, ${ }^{*}$ and Yuri Persidsky ${ }^{* \dagger}$
}

From the Department of Pathology and Laboratory Medicine* and the Center for Substance Abuse Research, ${ }^{\dagger}$ Lewis Katz. School of Medicine, Temple University, Philadelphia, Pennsylvania

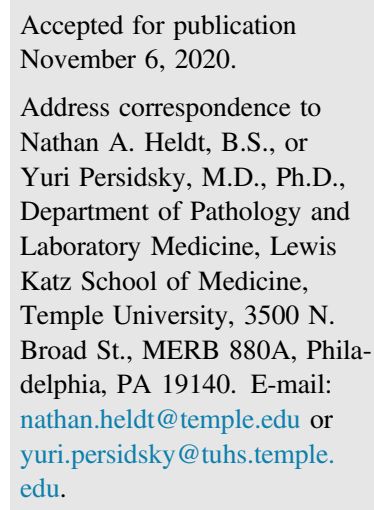

\begin{abstract}
Electronic nicotine delivery systems (often known as e-cigarettes) are a novel tobacco product with growing popularity, particularly among younger demographics. The implications for public health are twofold, as these products may represent a novel source of tobacco-associated disease but may also provide a harm reduction strategy for current tobacco users. There is increasing recognition that e-cigarettes impact vascular function across multiple organ systems. Herein, we provide a comparison of evidence regarding the role of e-cigarettes versus combustible tobacco in vascular disease and implications for blood-brain barrier dysfunction and cognitive decline. Multiple non-nicotinic components of tobacco smoke have been identified in e-cigarette aerosol, and their involvement in vascular disease is discussed. In addition, nicotine and nicotinic signaling may modulate peripheral immune and endothelial cell populations in a highly context-dependent manner. Direct preclinical evidence for electronic nicotine delivery system-associated neurovascular impairment is provided, and a model is proposed in which non-nicotinic elements exert a proinflammatory effect that is functionally antagonized by the presence of nicotine. (Am J Pathol 2021, 191: 243-255; https:// doi.org/10.1016/j.ajpath.2020.11.007)
\end{abstract}

Widespread availability of electronic nicotine delivery systems (ENDS; alias e-cigarettes) has ushered in a new era of youth tobacco use and continues to raise urgent questions in the realm of public health and policy. In 2014, ENDS overtook cigarettes as the most commonly used tobacco product by high school students in the United States. ${ }^{1}$ In the same year, use of any tobacco product by US high school students increased for the first time since 1998. Before this, rates had consistently trended downward, extending back to the adoption of youth tobacco reduction initiatives in the late 1990s. This inflection point is entirely attributable to ENDS, as all other tobacco product use has continued to decline. Furthermore, high school ENDS use in 2018 increased $77.8 \%$ over the previous year, representing the largest increase in youth tobacco use ever recorded by the
CDC. ENDS are used by $27.5 \%$ of US high school students, with similar increases in prevalence emerging internationally. $^{2-4}$ Although there is an inverse association between the age of individuals and their likelihood of ever using an ENDS, the prevalence of use in adults has also trended upward. Use estimates are much more divergent, but fell between $15 \%$ and $20 \%$ in 2015 to 2016 . $^{5}$

Little is known regarding the safety and the long-term health impact of these products. Despite a trend toward vaporized polysubstance use, the vast majority of products contain nicotine. 6 There are concerns that ENDSs may therefore be more addictive or increase the odds of future

Supported by NIH R01DA040619 (Y.P.) and T32DA007237 (N.A.H.). Disclosures: None declared. 
combustible cigarette use in adolescents. This concern has grown particularly urgent because of increasing concentrations of nicotine in recent products. ${ }^{7}$ The average concentration of nicotine has trended upward over time and is most notable in the case of Juul, in which the net nicotine content far exceeds that of cigarettes. Careful control over the fraction of protonated nicotine makes the delivery of more nicotine possible while minimizing the associated aversive effects. Changing the protonated fraction also impacts pharmacokinetics and sensory perceptions, potentially influencing addiction liability. ${ }^{8}$ In the arena of smoking cessation, ENDS may potentially represent a new tool for nicotine replacement that provides tactile and sensory cues similar to cigarettes. Whether they ultimately prove useful in this context will depend on their health profile balanced against their effectiveness in smoking reduction or cessation. The impact of ENDS on pulmonary function has garnered considerable attention, and both preclinical and clinical studies have begun to demonstrate airway inflammation and alterations in pulmonary immunity. ${ }^{9}$ Few studies directly address potential systemic (nonpulmonary) consequences, which are a significant contributor to tobaccoassociated morbidity ${ }^{10}$ and may have a more insidious onset in ENDS users. ENDS have a relatively short history of use and, therefore, preclinical research is necessary to provide insight regarding these long-term health care and public health questions.

\section{Literature Search Criteria}

Articles associated with this review were identified via PubMed search with publication dates beyond 2005 and online availability before April 20, 2020. The search terms used included the following: e-cigarette, electronic cigarette, vaping, vape, juul, juuling, smoking, cigarette, tobacco, nicotine AND requirement for inclusion of at least one of the following terms: vascular, vessel, blood-brain barrier, dementia. Criteria for screening included articles published in English in peer-reviewed journals and did not include case studies or series. Out of an abundance of caution and because of concerns about conflict of interest, studies that received tobacco industry funding were not considered. ${ }^{11}$ The scope of the present work includes studies of systemic vasculature that have neurovascular relevance, which required that studies dealing with exclusively pulmonary vascular effects be excluded. Lastly, analysis of literature prioritized clinical and in vivo evidence and included in vitro work only where experimental parameters had been extensively correlated with clinical conditions. Initial search and screening identified 298 articles, which were incorporated into the initial draft and reduced to 100 for the purposes of publication.

\section{ENDS Function and Constituents}

ENDS differ from existing tobacco products by use of a vaporization device (alias vape) to produce an inhalable aerosol. ${ }^{12}$ The more recent designs often allow customization of coil type, wicking material, and tank size, as well as user control over voltage. Changing these parameters can generate unique heating profiles that impact the composition of the aerosol. Furthermore, components of the vaporizer are in close contact with the aerosolized liquid (e-liquid) that the user consumes. On application of electric current and extreme heating, materials from the vaporizer have been found in the inhaled aerosol. ${ }^{12,13}$ Beyond nicotine, e-liquid formulations utilize propylene glycol, vegetable glycerin, or both as a base solvent and typically include one or more flavoring additives. There is an overabundance of flavoring products on the market. In 2014, there were an estimated 7764 unique flavors available from 466 brands. ${ }^{14}$ Far from being passive elements, some constituents have garnered special attention because of their acute toxicology ${ }^{15}$ or addiction-reinforcing properties. ${ }^{16}$

\section{Parallels to Cigarette Use}

When approaching the question of ENDS safety and human health, it is imperative that commonalities with combustible cigarettes are recognized. Decades of previous research regarding cigarette use may help to predict areas of concern, as well as highlight aspects of cigarette use that are absent in ENDSs. Nicotine is the most well-characterized constituent found in both products; however, many other substances, including reactive aldehydes, volatile organic compounds, metals, tobacco-specific nitrosamines, and reactive oxygen species (ROS), have been identified in e-liquids and aerosols. ${ }^{12}$ Furthermore, analysis of bronchoalveolar lavage fluid from ENDS users and smokers has identified some shared changes to the proteome, as well as findings that are exclusive to the ENDS group. ${ }^{17}$ ENDS exposure also appears to promote lipid accumulation in alveolar macrophages, a feature that is not observed following cigarette exposure and that appears to be nicotine-independent. ${ }^{18}$ Although pathology beyond the lung is unlikely to be clinically apparent because of the short history of use, mouse models of inhaled ENDS exposure have demonstrated enhanced atherosclerosis, cardiac dysfunction, and urothelial hyperplasia, ${ }^{19,20}$ mirroring known sequelae of long-term cigarette use. On the basis of these similarities in composition and biological impact, further consideration of potential shared hazards is warranted.

\section{Aerosolized Constituents}

Reactive aldehydes, such as formaldehyde, acetaldehyde, and acrolein, are generated at high levels by combustible cigarettes and represent a significant health hazard in smokers. They likely exert toxicity by multiple mechanisms, including formation of adducts with proteins and nucleic acids. ${ }^{21}$ It is generally agreed that ENDS expose users to lower levels of reactive aldehydes than do cigarettes, but the precise range of exposures is less clear. Acetone, 
formaldehyde, acetaldehyde, and propionaldehyde have been identified in aerosol from several ENDS brands, including the standardized research e-cigarette produced by the NIH. ${ }^{22}$ Concentrations of these compounds were between 5- and 50-fold below the level observed in smokers, and several studies failed to identify these emissions, ${ }^{12,23}$ possibly because of methodological limitations. Regardless of the debate surrounding aerosol aldehyde content, it is clear that ENDS users are exposed to more reactive aldehydes than nonusers under normal use conditions. Aldehyde metabolites are increased in the urine of ENDS users, although at lower levels than observed in cigarette smokers. ${ }^{24}$ Given the known association of aldehyde generation with higher device power ${ }^{25}$ and the trend toward increased power in newer generations of design, it is likely that ENDS users will be exposed to progressively higher aldehyde concentrations over time.

The deleterious effects of ROS contribute substantially to smoking-associated vascular disease. ${ }^{26}$ Direct measure of exogenous ROS presents technical challenges, but the presence of free radicals has been detected in ENDS aerosols using an adduct formation approach. ${ }^{22}$ Similar to reactive aldehydes, the generation of ROS is likely to vary considerably as a function of e-liquid content and vaporizer parameters. ${ }^{27}$ Although not a direct measure, the presence of low-density lipoprotein with greater susceptibility to oxidation in regular ENDS users would suggest a greater oxidative burden resulting from use. ${ }^{28}$

The insoluble particulate fraction of cigarette smoke, generally referred to as tar, is an impactful harm-associated component of smoke. The atherosclerotic potential of cigarette smoke has been partly tied to the particulate phase, and gene expression profiles in lung are altered as a function of particulate content. ${ }^{29}$ Although the chemical identity of the particulate is likely to be of importance, inhalation of particulate matter from a diversity of sources has been studied, including from secondhand tobacco, environmental pollution, and wildfires. Exposure to particulate has been linked to hypertension, myocardial infarction, heart failure, and cerebrovascular disease. ${ }^{30}$ Markers of vascular injury are also increased within cerebrospinal fluid as a function of fine particulate matter exposure. ${ }^{31}$ The net emission of particulate is lower than that of conventional smoke; however, the health impact of differing size profiles is unclear. Computational models indicate that deposition and absorption of particulate in the lungs of ENDS users may exceed those of cigarette smokers because of the smaller average particulate weight and diameter. $^{32}$

Several constituents of tobacco smoke are remnants of the Nicotinia tabacum plant and its processing, including tobacco-specific nitrosamines and the elemental metal cadmium. Despite demonstration of harm originating from these compounds in cigarette smoke, they have been identified in e-liquids and aerosols containing either nicotine or tobacco flavorings. The two compounds of highest abundance are 4-(methylnitrosamino)-1-(3-pyridyl)-1butanone (NNK) and $\mathrm{N}^{\prime}$-nitrosonornicotine (NNN), which contribute to oral, esophageal, and pulmonary oncogenesis. ${ }^{33} \mathrm{NNK}$ and NNN are abundant enough in cigarettes to pose a secondhand health risk through sidestream smoke and thirdhand deposition on surfaces.

Nicotine is by far the most investigated constituent of cigarettes and ENDS products, and has therefore been extensively characterized in relation to numerous organ systems. The primary determinant of daily ENDS use is nicotine content. Experienced users titrate daily use in relation to the nicotine percentage of provided e-liquid. ${ }^{34}$ This may mean that similar levels of nicotine, but higher levels of other toxicants, are introduced if former smokers attempt to wean nicotine content without limiting their daily ad libitum ENDS use. Patterns of nicotine exposure also fluctuate in a circadian manner similar to observations in smokers, with higher concentrations during the day and decreased but nonzero plasma levels overnight. ${ }^{35}$ Nicotine and its metabolites represent a common biomarker of tobacco product exposure for which analytical tools have been developed and clinical data have been collected. Given the paucity of specific biomarkers for ENDS use, ${ }^{36}$ quantification of nicotine and its major metabolite, cotinine, may represent the best available means for development and standardization of research models.

\section{Macrovascular Impact}

Beyond the impact of acute ENDS exposure on pulmonary function, considerable attention has centered on macrovascular consequences (Figure 1). Ongoing use of combustible tobacco leads to an increased risk of numerous cardiovascular diseases, including coronary heart disease, stroke, and peripheral artery disease. ${ }^{37}$ Risk for these conditions is mediated in part by smoke-mediated endothelial dysfunction. Exposure to smoke triggers production of ROS through the NADPH oxidases, causes release of NF- $\kappa \mathrm{B}$, and reduces availability of nitric oxide, leading to reduced flowmediated dilation. ${ }^{38}$ Further measures of endothelial damage in smokers include the release of von Willebrand factor, progenitor cells, and endothelial microparticles into circulation. ${ }^{39-41}$ Considering the ability of nicotine to reduce endothelial nitric oxide synthase in endothelial cells, ${ }^{42}$ there is reason to believe that alternative products for nicotine delivery, such as ENDS, may confer the same risks.

The physiological effects of acute ENDS exposure have been examined in several small studies that suggest commonalities with cigarette use. Regardless of nicotine content, use of ENDS appears to elevate systolic and diastolic blood pressure. Other parameters, such as heart rate and sympathetic predominance, appear to be nicotine-dependent. Heart rate is elevated following use of nicotinized but not nicotine-free ENDS, and measurements of heart rate variability indicate sympathetic predominance, specifically in nicotine-containing products. ${ }^{43,44}$ Sympathetic predominance 


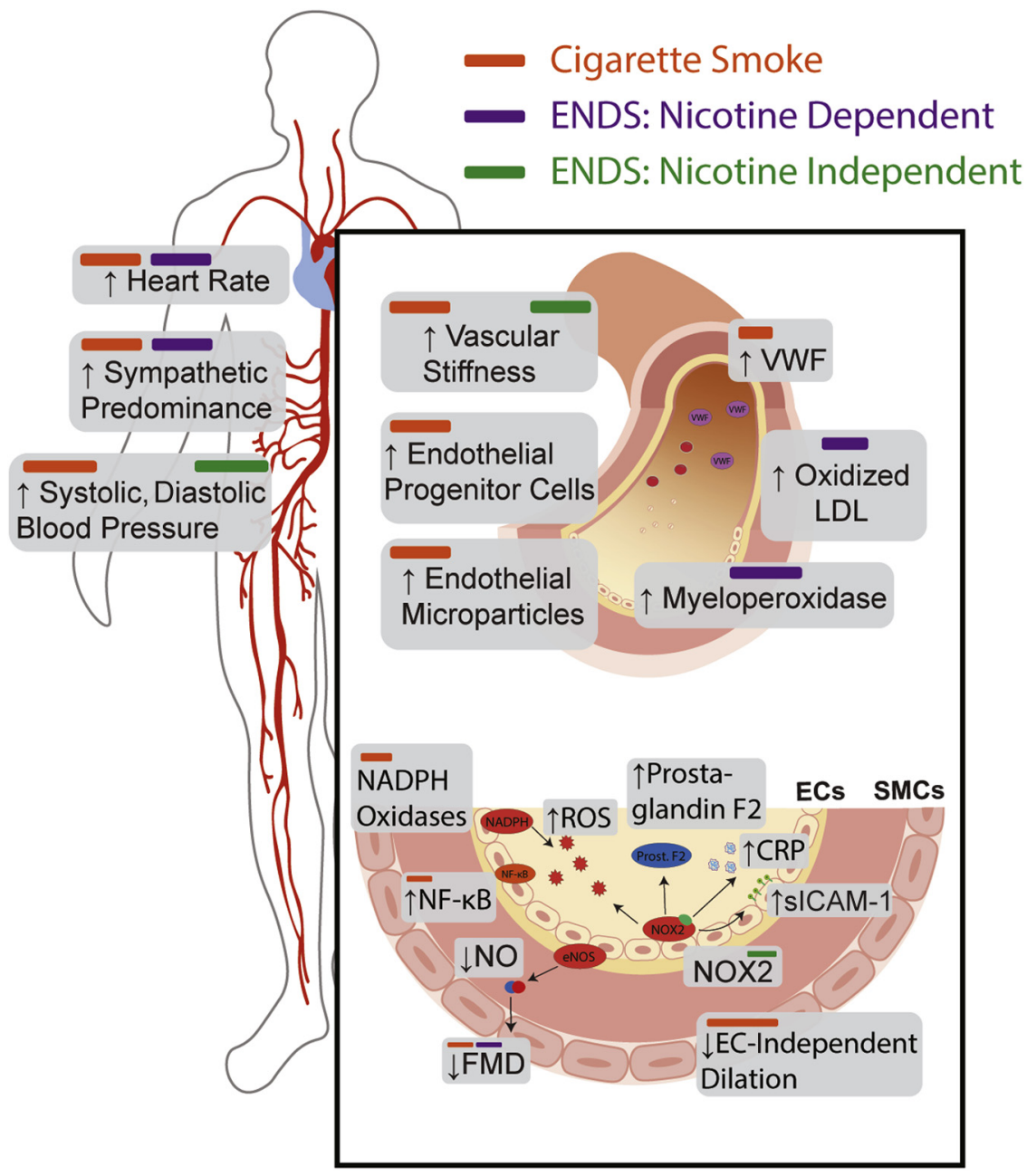

Figure 1 Summary of evidence demonstrating the macrovascular consequences of combustible cigarette versus electronic nicotine delivery system (ENDS) use. Physiological measures of vascular function and serum inflammatory markers are impacted following ENDS or cigarette use. Specifically, heart rate and blood pressure are increased, the sympathetic nervous system becomes dominant, flow-mediated dilation (FMD) is decreased, and there is increased vascular stiffness. With the exception of blood pressure and vascular stiffness, all of these outcomes appear to be nicotine dependent. The scope of molecular investigations often does not cover both products, but some similarities are observed. Reactive oxygen species (ROS) are generated by both products in a nicotineindependent manner, and this is specifically a result of cytochrome b-245 heavy chain (NOX2) activity in the case of ENDS. Circulating biomarkers have been identified in both cases, although the investigated markers are unique for cigarettes [von Willebrand factor (VWF), endothelial progenitor cells, and endothelial microparticles] versus ENDS [oxidized low-density lipoprotein (LDL) and myeloperoxidase]. CRP, C-reactive protein; EC, endothelial cell; eNOS, endothelial NO synthase; NO, nitric oxide; sICAM-1, soluble intracellular adhesion molecule 1; SMC, smooth muscle cell.

is particularly important because it is predictive of numerous cardiovascular diseases. However, smokers who are able to transition to exclusive ENDS use exhibit smaller increases in these parameters compared with continued cigarette use. ${ }^{45}$ This suggests that non-nicotinic constituents of combustible cigarettes also contribute to this effect. In terms of endothelial function, measurements of flow-mediated dilation are informative because of the role of endothelial cells in sensing increased flow and mediating vasodilation by increasing nitric oxide through endothelial nitric oxide synthase. Flowmediated dilation response is blunted regardless of nicotine content. ${ }^{46}$ Side-by-side comparisons with cigarettes demonstrate a lower flow-mediated dilation impairment compared with nicotinized ENDS. Measures of nitric oxide bioavailability also support the conclusion that ENDS are less harmful to endothelial function. ${ }^{47}$ Vascular stiffness is also increased by ENDS in a nicotine-independent manner, although to a lesser extent than with cigarettes. ${ }^{43,48}$ Interestingly, involvement of cytochrome b-245 heavy chain (alias NOX2) was supported by two separate studies. In one study, NOX2 contributed to the elevation of prostaglandin F2; and in a separate investigation, it facilitated the elevation of circulating
C-reactive protein and soluble intercellular adhesion molecule 1 (ICAM-1) and increased ROS. ${ }^{47,49}$ The effect of NOX2 also appeared to be independent of nicotine.

The presence of nicotine in ENDS increases vascular dysfunction and inflammatory markers acutely, although the effects are of lesser magnitude when compared with similar nicotine dosing through combustible cigarettes. Acetylcholine-mediated vasodilation occurs through direct action at smooth muscle cells and does not require modulation by endothelial cells. This parameter is unchanged in current smokers when exposed to nicotine-free ENDS, but is significantly blunted following exposure to an equivalent product with nicotine. ${ }^{50}$ Although not organ system specific, an increase in circulating myeloperoxidase was also observed exclusively following nicotinic ENDS exposure. Given the elevated concentration of ENDS constituents specifically present within the lung, it is possible that circulating myeloperoxidase is primarily attributable to pulmonary neutrophils. Although many cardiovascular alterations are observed following both acute cigarette and ENDS use, carbon monoxide exposure does not occur with ENDS use. ${ }^{36}$ 
The short history of ENDS use has limited the scope of conclusions that can be drawn regarding chronic use, but some trends have begun to emerge. The sympathetic predominance observed following acute ENDS exposure persists regardless of acute abstinence in long-term ENDSs. In a cohort of ENDS users with an average of 1.6 years of use, heart rate variability was decreased, indicating sympathetic predominance. Oxidized low-density lipoprotein was also elevated. ${ }^{28}$ The ongoing sympathetic predominance may be an early indicator of increased cardiovascular events in current ENDS users. Indeed, case-control data drawn from a national survey indicate elevated rates of myocardial infarction associated with everyday use. ${ }^{51}$ Dual use with cigarettes increases risk of coronary heart disease, myocardial infarction, or stroke by $36 \%$, compared with use of cigarettes alone. ${ }^{52}$ Although the conclusion of increased cardiovascular risk appears definitive on the basis of these data, potential confounding by former cigarette use must be considered. More than half of the ENDS group studied by Moheimani et $\mathrm{al}^{28}$ were former cigarette users compared with $11 \%$ of controls. Given the strong association between former or current cigarette use and current ENDS use in adult populations, further prospective studies are needed before this conclusion can be reached.

\section{The Blood-Brain Barrier}

The cerebral microvasculature is a highly specialized vascular bed with duties that extend beyond the vessels of most other organs. The extracellular environment within the central nervous system must be tightly regulated to facilitate normal neural function. Passage of molecules and pathogens is highly restricted, and it is classically described as immune privileged, meaning that it is not subject to surveillance by peripheral immune cells. The collection of specialized cells and structures facilitating this, known as the blood-brain barrier (BBB), include endothelial cells, pericytes, a basal lamina, and astrocytic end feet that completely encircle the vessel. Broadly speaking, functions of the BBB may be classified into physical, transport, and metabolic.

Entry into the central nervous system requires that several physical barriers be traversed, including highly specialized endothelial cells that lack both fenestrations and pinocytosis, limiting transcellular permeability. Paracellular transit is also limited primarily by the presence of tight junction (TJ) complexes between endothelial cells, which are anchored to cytoskeletal elements intracellularly and connect adjacent cells through extracellular domains. Loss of physical barrier integrity can be associated with transcellular or paracellular routes, and independent dysfunction of each has been demonstrated in several disease states. ${ }^{53,54}$ The transmembrane components of TJ complexes consist primarily of claudins-1, -3 , and -5 (CLDN1, CLDN3, and CLDN5, respectively) as well as occludin, junctional adhesion molecules, and endothelial cell-selective adhesion molecule. ${ }^{55}$ These components interface with elements of the cytoskeleton via the adapter tight junction proteins $\mathrm{ZO}$ 1, -2 , and -3 (TJP1, TJP2, and TJP3, respectively). More importantly, the strength of the physical barrier may be modulated by interaction with circulating peripheral immune cells or cytokines. Engagement by HIV-infected monocytes increases permeability through the Rho-dependent phosphorylation of TJPs, and permeability can also be increased by direct C-X-C chemokine receptor type 4 or C$\mathrm{C}$ chemokine receptor type 5 agonism at endothelial cells ${ }^{56,57}$ Considered in whole, it is clear that although the physical barrier of the BBB is directly attributable to endothelial cells and the integrity of their TJ complexes, it is modulated by peripheral immune cells, circulating factors (generally proinflammatory), and other components of the neurovascular unit.

Despite a lack of passive diffusivity across the neurovascular interface, the central nervous system has the highest metabolic demand of any organ and requires roughly $20 \%$ of available blood flow. This apparent paradox highlights the importance of barrier transporters for clearance of waste and delivery of hydrophilic nutrients, such as glucose and amino acids. The entry of glucose is primarily facilitated by solute carrier family 2, facilitated glucose transporter member 1 (GLUT1) and solute carrier family 2, facilitated glucose transporter member 3 (GLUT3). ${ }^{55}$ These transporters are known to respond to a variety of physiological and pathologic conditions, including hypoglycemia, cerebral ischemia, and Alzheimer disease (AD). ${ }^{58,59}$ Decreased glucose transport in $\mathrm{AD}$ patients has been linked to decreased levels of GLUT1 and GLUT2 expression while mRNA levels remained constant, presumably indicating increased turnover as a culprit. Beyond the active transport of passively insoluble nutrients, the BBB exhibits greater impenetrability to lipophilic substrates than would be predicted on the basis of passive measures alone. This is attributable to the expression of drug efflux pumps, such as ATP-dependent translocase ABCB1 (alias P-gp), multidrug resistance-associated protein 1 (alias MRP1), and broad substrate specificity ATP-binding cassette transporter ABCG2 (alias BCRP) ${ }^{55}$ Although serving an evolutionarily beneficial purpose through efflux of infectious and metabolic toxins, the transport function of the BBB is often an impediment to drug development.

In addition to nutrient transport and efflux roles, the BBB must also facilitate the appropriate transit of cells and macromolecules that are beyond the capacity of normal transmembrane transporters. In the case of many large molecules, this occurs via receptor-mediated transcytosis. Interestingly, many inflammatory and immune-associated molecules are transited across the BBB by this mechanism, including tumor necrosis factor (TNF), immunoglobulins, and receptor for advanced glycosylation end product-specific receptor (alias RAGE). ${ }^{55}$ In addition, activated peripheral immune cells may induce a proinflammatory endothelial cell state that facilitates their passage into brain parenchyma. This is accompanied by up-regulation of selectins, ICAM-1, and 
vascular cell adhesion protein 1 (VCAM-1), and results in elevated paracellular permeability via reduction in TJ integrity. ${ }^{60,61}$ Overall, the transport functions of the BBB generate a stable environment for neurologic function under physiological conditions but can become compromised and further propagate proinflammatory signaling under pathologic conditions.

\section{Smoking, Cognitive Impairment, and Cerebrovascular Disease}

The association between smoking history, cerebrovascular disease, and cognitive impairment has been somewhat controversial. The earliest epidemiologic studies examining smoking as a potential risk factor for AD identified both positive and negative associations, whereas those based purely on post-mortem pathology reported a net protective effect. ${ }^{62,63}$ Research conducted since then led to the consensus that $\mathrm{AD}$ risk is increased, with an estimated relative risk of 1.72 in current smokers. ${ }^{64}$ The discrepancy with early study outcome has been attributed to the predominant use of retrospective case-control studies rather than a prospective approach; however, there is ongoing speculation that nicotine alone may improve histopathologic correlates in $\mathrm{AD}$ animal models. ${ }^{65}$

Although AD was the first type of dementia linked to smoking and has historically been thought of as a distinct etiology, it is now recognized that most cognitive decline is more often the result of multiple pathologic processes. On the basis of autopsy studies, roughly half of cases positive for $\mathrm{AD}$ pathology also have findings associated with other forms of dementia. ${ }^{66}$ These findings often include cerebral amyloid angiopathy associated with vascular dementia (VD), the second most common form of dementia worldwide following AD. Cerebrovascular lesions are found in between $50 \%$ and $84 \%$ of individuals who live past 80 years of age, ${ }^{67}$ and signs of VD on imaging and post-mortem examination often go undetected, so it is likely that prevalence of pure VD and its coexistence with $\mathrm{AD}$ are underestimated. Despite the lack of studies directly examining associations between VD and smoking, it is clear that behaviors and risk factors are nearly identical between $\mathrm{AD}$ and $\mathrm{VD} .{ }^{68}$ In addition, the early pathogenesis of $\mathrm{AD}$ is thought to include microvascular dysfunction and BBB breakdown, including pericyte loss and deficits in amyloid- $\beta$ transcytosis. ${ }^{69}$ In light of these developments and considering the wellestablished relationship between smoking and microvascular dysfunction, it is reasonable to conclude that microvascular dysfunction is a contributing factor to smoking-associated cognitive decline.

When cognitive function is considered independent of the underlying pathology, it is clear that a long-term history of smoking predisposes to worsened cognition. In middle-aged chronic smokers, performance was worse on a battery of cognitive tests assessing cognitive flexibility, speed of cognitive processes, and memory. ${ }^{70}$ In addition to performing poorly at baseline, follow-up assessment after 5 years showed a greater rate of decline in smokers compared with nonsmokers, and overall performance was significantly associated with smoking history in pack-years. Although these findings are likely to be subclinical during middle age, smokers beyond 60 years begin to demonstrate alterations on brain imaging and clinical signs of dementia. White matter hyperintensities on magnetic resonance imaging are associated with BBB dysfunction and often indicate some level of vascular cognitive impairment. ${ }^{71}$ Smokers have an elevated volume of white matter hyperintensities and reduced total white matter volume, ${ }^{72}$ suggesting worsening microvascular function in the brain. White matter lesions can also be inversely correlated with overall cognitive functioning and processing speed in the same cohort of patients. In addition, the relative risk for development of all forms of dementia is 1.27 in current smokers, including a 1.78 incident risk for VD. ${ }^{73}$ Despite these robust data linking cigarette use to impaired cognition and dementia, current knowledge regarding the causative constituents or the mechanism by which they act is lacking.

\section{Contribution of Nicotinic Signaling}

Nicotine exerts its effects on biological function through nicotinic acetylcholine receptors, and nicotinic signaling is likely to explain a substantial portion of ENDS impact on human physiology. Functional nicotinic receptors are composed of five symmetric subunits surrounding a central ion pore. ${ }^{74} \alpha 7$ homomeric nicotine acetylcholine receptors $(\alpha 7 \mathrm{nAChRs})$ are of greatest relevance in endothelial and immune function and are encoded by the CHRNA7 gene in humans. Notably, the human genome also includes a partial duplication known as CHRFAM7A, which has a dominantnegative influence when expressed. The expression balance of these isoforms is of particular relevance in peripheral tissues of tobacco users, as it appears that CHRFAM7A is expressed basally but CHRNA7 is induced exclusively in monocytes from smokers. ${ }^{75}$ This variation does appear to have functional consequences, as monocytes from smokers differ in their response to cholinergic signaling in an inflammatory context. Overall, the widespread expression of $\alpha 7 \mathrm{nAChRs}$ across vascular and immune tissue makes them a likely mediator for the nonneural actions of nicotine.

\section{Immune Cells}

In contrast to a known cytotoxic effect at concentrations observable in the lung, nicotine may exhibit antiinflammatory effects at the lower concentrations present peripherally. This has been demonstrated preclinically in several acute and chronic disease conditions. ${ }^{76}$ Levels of type 1 helper $\mathrm{T}$ cell-associated cytokines, such as TNF, IL-1 $\beta$, IL-6, and IL- 
$12 \beta$, were reduced in the plasma of rat preeclampsia models that had been induced with lipopolysaccharide and pentylenetetrazol. Similarly, nicotine administration in a mouse model of sepsis prevented the release of high-mobility group protein $\mathrm{B} 1$ and inhibited NF- $\kappa \mathrm{B}$ activation specifically through $\alpha 7$ nAChRs. ${ }^{77}$ This phenomenon is also generalizable to chronic inflammatory conditions, as TNF was reduced by nicotine administration in disease models that included inflammatory bowel disease. ${ }^{78}$ Myeloperoxidase activity, leukotriene production, and plasma corticosterone were also decreased by nicotine in the context of inflammatory bowel disease. It is likely that the endogenous function of such a mechanism is to serve a larger homeostatic pathway, such as the cholinergic antiinflammatory pathway. ${ }^{79}$ This pathway facilitates cross talk between nervous and immune networks through a vagal reflex. Modulation of cytokine production plays a central role, in part through the direction of T-cell differentiation into specific lineages. In this context, nicotinic signaling may act to shift the immune response toward an alternative type 2 helper T-cell state of activation rather than a global down-regulation of all proinflammatory elements.

Despite the largely antiinflammatory effect observed in animal models, the immunomodulatory aspects of nicotine are less clear in the clinical realm. Several studies have examined the role of nicotine in a variety of autoimmune diseases, including Behçet disease, sarcoidosis, and inflammatory bowel disease. ${ }^{80}$ Taken as a whole, the evidence from inflammatory bowel disease suggests that the net effect of nicotine is highly location-dependent, with exacerbation of disease in small bowel and beneficial effects largely confined to the colon. The evidence associated with Behçet disease is less compelling, but may suggest similar phenomena. Nicotine patches were effective in addressing cutaneous symptoms and mucosal ulcerations, but did not alleviate manifestations within the joint or eye. These findings suggest that there may be some value to the inclusion of nicotine in e-liquid formulations, but also demonstrate that the effect is highly dependent on the organ system and immune status of the individual. The impact of nicotine on human immune function has not been studied adequately in disease-free individuals, and mechanistic studies raise some theoretical concerns. Although there is evidence for regulatory T-cell activation and a net antiinflammatory effect in some contexts, ${ }^{80}$ the possibility of nicotine-associated type 2 helper T-cell induction ${ }^{79}$ may be of concern for individuals predisposed to atopy. A more thorough clinical understanding of nicotinic immunomodulation is needed to understand its potential role in e-liquid formulations.

\section{Endothelium}

The impact of nicotine on nonpulmonary endothelium is highly context-dependent, with outcomes largely based on the context and duration of exposure. Endothelial effects are mediated primarily through $\alpha 7 \mathrm{nAChRs,}$ which, in nonneural cells, are capable of activating several metabotropic pathways in addition to their ionotropic role. In human umbilical vein endothelial cells, treatment with nicotine induced expression of the adhesion molecules ICAM- 1 and VCAM- $1{ }^{81}$ This was mediated by sequential signaling through p38 mitogen-activated protein kinases and protein kinase $\mathrm{C}$ to increase NF- $\mathrm{BB}$ and AP1 activity, and blockade at any of the intermediate signaling points prevented subsequent increases in adhesion molecules. Time points of longer duration within the same study indicate that this apparent proinflammatory effect is attenuated over time, possibly as a result of contributing ionotropic currents and receptor desensitization. ${ }^{81,82}$ In addition, signaling in endothelial cells can also occur through JAK2-STAT3 ${ }^{83}$ In both macrovascular and microvascular cells, nicotinic signaling reduced expression of ICAM-1 and decreased C-C motif chemokine 2 production in a JAK2STAT3-dependent manner following administration of IL-6. Further evidence for a generalizable antiinflammatory influence on endothelium when paired with inflammatory stimuli is evident in vivo. Pretreatment with nicotine and cholinergic agonists prevents TNF-induced mRNA expres-

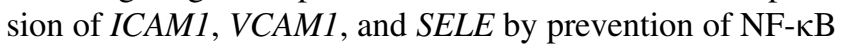
translocation and reduces leukocyte migration in the carrageen air pouch model. ${ }^{84}$ Similar effects on endothelial response could be seen when nicotine or cholinergic agonists were combined with IL-6, lipopolysaccharide, and in the physiological effects of pregnancy. ${ }^{83,85,86}$ In aggregate, these findings suggest that endothelial exposure to nicotine under basal conditions may lead to transient inflammation, but also may attenuate endothelial response to inflammatory cytokines and other stimuli.

\section{Cerebrovascular Features}

Although specific nicotinic effects at the BBB are poorly characterized, the previously described work in other endothelial cell types is likely to have some applicability. A small number of studies have investigated BBB endothelium directly. Within bovine brain microvessel endothelial cells, exposure to high levels of nicotine or cotinine is known to decrease expression of TJP1 and consequently increase paracellular permeability. ${ }^{87}$ The prior addition of $\alpha$-bungarotoxin, an $\alpha 7$ antagonist, negates this effect, demonstrating that it is $\alpha 7 \mathrm{nAChR}$-dependent. Furthermore, the expression of both neuronal acetylcholine receptor subunit $\alpha 7$ and $\beta 2$ subunits was decreased in cells exposed to nicotine or cotinine. Increased permeability could similarly be observed in rats following continuous administration of nicotine by osmotic minipump ${ }^{88}$ and was associated with localization deficits in TJP1 and CLDN3, although total protein levels were unaffected. TJ deficits and increased paracellular permeability are both strong correlates of inflammation in cerebral endothelium, and it is possible that these observations coincide with $\mathrm{p} 38$ mitogen- 
activated protein kinase and protein kinase $\mathrm{C}$ signaling, which promotes inflammation in other endothelial cell types. However, concentrations and dosing of nicotine in these studies exceeded the maximum peak observable in the plasma of human smokers. ${ }^{36}$ Human dosing also fluctuates with circadian rhythm, and it is unclear whether changing these parameters substantially modifies the effect. In contrast, BBB integrity and expression of claudins can be preserved in the context of intracerebral hemorrhage by agonism of $\alpha 7 \mathrm{nAChR} .{ }^{89}$ In this context, the protective effect occurs due to inhibition of glycogen synthase kinase$3 \beta$ through the phosphatidylinositol 3-kinase-Akt pathway, reducing turnover of catenin $\beta 1$. Taken together, these data suggest multiple modes of signaling for $\alpha 7$ nAChR that may contribute to basal inflammation but also dampen the response to existing inflammatory stimuli. To further complicate matters, the effect of $\alpha 7 \mathrm{nAChRs}$ in culture appears to be highly cell-type-dependent. Recent evidence from primary rat brain endothelial cells shows upregulation of CLDN5 and occludin as well as decreased permeability to sodium fluorescein following $\alpha 7$ agonism. $^{90}$ This is an apparent contradiction to earlier work $^{87,88}$ and highlights the need for further studies to clarify the role of nicotine in the context of modern tobacco product use.

\section{ENDS-Induced Microvascular Dysfunction}

There is an urgent need to assess the risk of protracted ENDS use by available preclinical tools. A small number of studies provide an initial framework for investigating the global effects of ENDS outside the lung. The earliest nonpulmonary in vitro studies utilizing whole ENDS components took an infusion approach, comprising vaporization of various e-liquids followed by diversion of the aerosol through cell culture media. ${ }^{91}$ Following treatment of liver Kupffer cells with ENDS or cigarette smoke extracts, comparable increases in complement deposition as well as elevated expression of complement receptors were observed. Notably, increasing concentrations of nicotine appear to dampen the proinflammatory response to ENDS extracts by some measures, including $\mathrm{C} 4 \mathrm{~d}$ deposition and complement component $\mathrm{C} 1 \mathrm{q}$ receptor (CD93) expression. This is congruent with the fact that markers of inflammation, oxidative stress, and lipid peroxidation are elevated in mice exposed to nicotine-free aerosol formulations versus nicotine-containing counterparts. ${ }^{92}$ It is thought that this may be mediated by a CYBBNOX2-dependent mechanism initiated via inhaled ROS and aldehydes and antagonized by nicotinic signaling. These observations are particularly interesting when considered alongside mounting evidence that the presence of nicotine in proinflammatory contexts may, in fact, moderate the degree of inflammation. The idea that tobacco products contain a cocktail of constituents with individually opposed actions is not a new one, as cigarette smoke is known to contain numerous proinflammatory and antiinflammatory compounds with sometimes paradoxical effects. $^{93}$ On this basis, it may even be postulated that nicotine serves to counteract the proinflammatory action of other e-liquid constituents, and therefore e-liquids that are unchecked by nicotinic influence may exert a more vigorous proinflammatory effect.

The neurovascular applicability of this concept is further supported by studies of the BBB in an inhalalation mouse model. Under conditions that mirror clinical parameters of use, we have recently demonstrated that ENDS exert dual actions on BBB function. ${ }^{94}$ Following months-long repeated exposure, a proinflammatory phenotype persisting beyond clearance of constituents from circulation was identified. Proteins, such as occludin and SLC2A1GLUT1, were uniformly reduced across cigarette and ENDS groups regardless of nicotine status, but functional consequences, such as increased permeability, were only observed following nicotine-free ENDS exposure. Similarly, working memory deficits were observed only in the nicotine-free group, and TNF-evoked leukocyte adhesion was greatest in this group as well.

Although counterintuitive, the contribution of nicotine is further clarified by recent work that combined acute episodes of ENDS exposure with classic inflammatory mediators (IL-1 $\beta$ and lipopolysaccharide). Following single episodes of exposure, ENDS alone had a negligible impact on inflammation and BBB function. However, preexposure to nicotine-containing formulations served to disrupt the response to subsequent inflammatory stimuli. Inflammationinduced BBB compromise and leukocyte recruitment were of lower magnitude when they immediately followed ENDS exposure. These functional findings were accompanied by ENDS-associated decreases in the expression of proinflammatory genes within systemic immune elements and brain microvasculature.

After examining the commonalities between available literature and our own findings, a working model of ENDS exposure at neurovascular sites can be proposed (Figure 2). Direct impact is negligible following acute exposure in naïve individuals. However, the delivered nicotine exerts an immunomodulatory role that attenuates the response of circulating leukocytes and endothelial cells to subsequent inflammatory stimuli. As bouts of exposure are repeated over months and years in the form of regular use, constituents such as reactive aldehydes, ROS, and particulate matter, begin to play a role. It is likely that the extent of exposure is constant over time, but the gradual depletion of antioxidant reserves and weakening of other defenses account for this increased magnitude of effect. The role of these constituents has been universally described as proinflammatory ${ }^{92,95}$ and begins to have lasting impact on neurovascular function. However, the immunomodulatory action of nicotine appears to be present even following ongoing exposure, and where present may be responsible for reducing functional consequences of ENDS and cigarette exposure at chronic time points. 


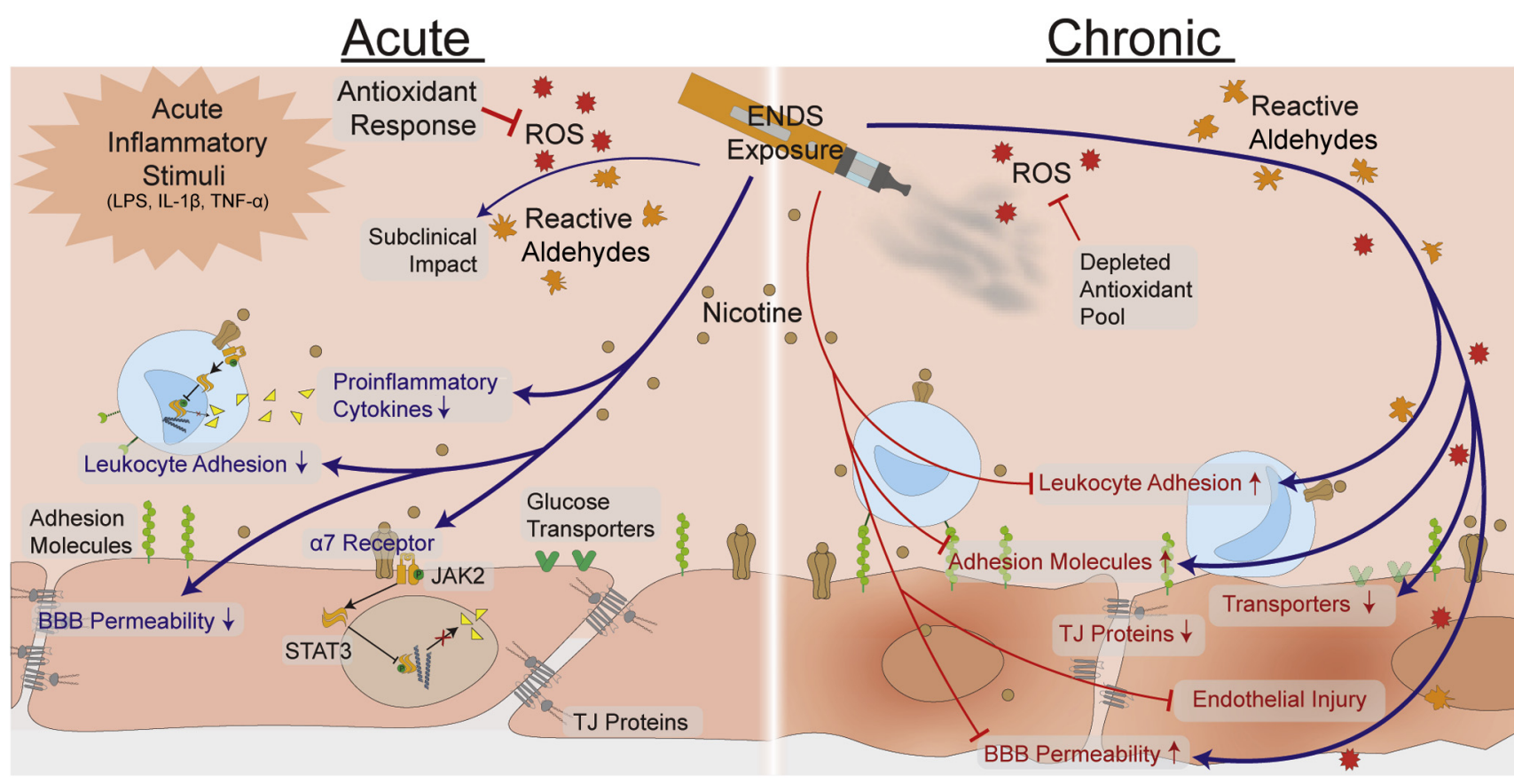

Figure 2 Electronic nicotine delivery systems (ENDS) exert dual action on the blood-brain barrier (BBB) following acute versus chronic use. Left panel: Acutely proinflammatory components, such as reactive oxygen species (ROS) and reactive aldehydes, have a largely subclinical impact because of strong antioxidant response. Nicotine has an immunomodulatory role when combined with inflammatory stimuli, which results in reduced leukocyte adhesion, lower expression of proinflammatory cytokines, and preserved physical barrier function. This is likely mediated through $\alpha 7$ homomeric nicotine acetylcholine receptor and JAK2-STAT3 signaling. Right panel: Following chronic use, antioxidants are depleted and the accumulation of ROS and reactive aldehydes produces a net proinflammatory effect, resulting in increased leukocyte adhesion, decreased tight junction (TJ) proteins and transporters, and increased BBB permeability. Nicotine continues to have an antiinflammatory role, which may functionally antagonize the proinflammatory elements of exposure. Blue arrows, induction; red arrows, inhibition. LPS, lipopolysaccharide; TNF- $\alpha$, tumor necrosis factor- $\alpha$.

\section{Contribution of Oxidative Stress}

The generation of ROS due to e-liquid vaporization and as a response to inhaled constituents occurs in both cigarette and ENDS use, and preclinical studies have demonstrated an association with neurovascular impairment and BBB

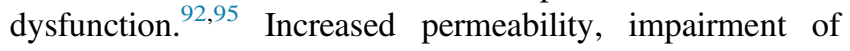
glucose transport, and greater susceptibility to subsequent insult are possible sequelae of ROS, and occur because of a depletion of endogenous antioxidant responses, such as nuclear factor erythroid 2-related factor 2-antioxidant response element (NFE2L2-AIRE) pathway and disruption of mitochondrial oxidation-reduction. ${ }^{96-98}$ Signaling through NFE2L2 evokes an endothelial inflammatory response, ${ }^{99}$ leading to the loss of TJP1 and induction of adhesion molecules, platelet endothelial cell adhesion molecule, ICAM-1, and VCAM-1. It would appear that endogenously produced ROS are a significant contributor to this mechanism, as both pharmacologic and genetic inhibition of CYBBNOX2 leads to a decrease in ROS following ENDS exposure in mouse models. ${ }^{92}$ In addition, tobacco-associated ROS can be attenuated via treatment with oral hypoglycemic agents, including metformin and rosiglitazone, which appear to act through induction of NFE2L 2. ${ }^{95,100}$ These studies strongly support the involvement of antioxidant response pathways in
BBB response to ENDS and may warrant further investigation in the clinical realm.

\section{Conclusions}

Collectively, the literature summarized herein indicates that multiple areas of overlap exist between ENDS and combustible tobacco. The known associations of ROS, reactive aldehydes, particulate, metals, and select flavorants with tobacco-related pathology raise concerns regarding the long-term sequelae of ENDS use. Microvascular dysfunction and cognitive decline are common in the smoking population and nearly ubiquitous in the late stages of tobacco-associated disease. It is also apparent that ENDS aerosols have the potential to impact vascular and immune function in an unpredictable manner. Individually known components do not behave in a summative way, necessitating characterization of whole products under naturalistic conditions. Furthermore, available evidence suggests that endothelial function is impacted, and cerebrovascular integrity is of particular concern. Given these facts, a comprehensive investigation of ENDS and their impact on BBB function and neuroinflammation is warranted. Ultimately, the future relationship between ENDS and 
public health cannot be determined without comprehensive, long-term assessment of the impact of ENDS on vascular function.

\section{References}

1. Gentzke AS, Creamer M, Cullen KA, Ambrose BK, Willis G, Jamal A, King BA: Vital signs: tobacco product use among middle and high school students - United States, 2011-2018. MMWR Morb Mortal Wkly Rep 2019, 68:157-164

2. Czoli CD, Hammond D, White CM: Electronic cigarettes in Canada: prevalence of use and perceptions among youth and young adults. Can J Public Health 2014, 105:e97-e102

3. de Lacy E, Fletcher A, Hewitt G, Murphy S, Moore G: Crosssectional study examining the prevalence, correlates and sequencing of electronic cigarette and tobacco use among 11-16-year olds in schools in Wales. BMJ Open 2017, 7:e012784

4. White J, Li J, Newcombe R, Walton D: Tripling use of electronic cigarettes among New Zealand adolescents between 2012 and 2014. J Adolesc Health 2015, 56:522-528

5. Levy DT, Yuan Z, Li Y, Mays D, Sanchez-Romero LM: An examination of the variation in estimates of e-cigarette prevalence among U.S. adults. Int J Environ Res Public Health 2019, 16:E3164

6. Romberg AR, Miller Lo EJ, Cuccia AF, Willett JG, Xiao H, Hair EC, Vallone DM, Marynak K, King BA: Patterns of nicotine concentrations in electronic cigarettes sold in the United States, 2013-2018. Drug Alcohol Depend 2019, 203:1-7

7. Omaiye EE, McWhirter KJ, Luo W, Pankow JF, Talbot P: Highnicotine electronic cigarette products: toxicity of JUUL fluids and aerosols correlates strongly with nicotine and some flavor chemical concentrations. Chem Res Toxicol 2019, 32:1058-1069

8. Ferris Wayne G, Connolly GN, Henningfield JE: Brand differences of free-base nicotine delivery in cigarette smoke: the view of the tobacco industry documents. Tob Control 2006, 15:189-198

9. Gotts JE, Jordt S-E, McConnell R, Tarran R: What are the respiratory effects of e-cigarettes? BMJ 2019, 366:15275

10. Centers for Disease Control and Prevention (US): How Tobacco Smoke Causes Disease: The Biology and Behavioral Basis for SmokingAttributable Disease: A Report of the Surgeon General [Internet]. Atlanta, GA, Centers for Disease Control and Prevention (US), 2010

11. Pisinger C, Godtfredsen N, Bender AM: A conflict of interest is strongly associated with tobacco industry-favourable results, indicating no harm of e-cigarettes. Prev Med 2019, 119:124-131

12. Dinakar C, O'Connor GT: The health effects of electronic cigarettes. N Engl J Med 2016, 375:1372-1381

13. Zhao D, Navas-Acien A, Ilievski V, Slavkovich V, Olmedo P, Adria-Mora B, Domingo-Relloso A, Aherrera A, Kleiman NJ, Rule AM, Hilpert M: Metal concentrations in electronic cigarette aerosol: effect of open-system and closed-system devices and power settings. Environ Res 2019, 174:125-134

14. Zhu S-H, Sun JY, Bonnevie E, Cummins SE, Gamst A, Yin L, Lee M: Four hundred and sixty brands of e-cigarettes and counting: implications for product regulation. Tob Control 2014, 23(Suppl 3): iii3-iii9

15. Allen JG, Flanigan SS, LeBlanc M, Vallarino J, MacNaughton P, Stewart JH, Christiani DC: Flavoring chemicals in e-cigarettes: diacetyl, 2,3-pentanedione, and acetoin in a sample of 51 products, including fruit-, candy-, and cocktail-flavored e-cigarettes. Environ Health Perspect 2016, 124:733-739

16. Avelar AJ, Akers AT, Baumgard ZJ, Cooper SY, Casinelli GP, Henderson BJ: Why flavored vape products may be attractive: green apple tobacco flavor elicits reward-related behavior, upregulates nAChRs on VTA dopamine neurons, and alters midbrain dopamine and GABA neuron function. Neuropharmacology 2019, 158:107729

17. Ghosh A, Coakley RC, Mascenik T, Rowell TR, Davis ES, Rogers K, Webster MJ, Dang H, Herring LE, Sassano MF,
Livraghi-Butrico A, Van Buren SK, Graves LM, Herman MA, Randell SH, Alexis NE, Tarran R: Chronic e-cigarette exposure alters the human bronchial epithelial proteome. Am J Respir Crit Care Med 2018, 198:67-76

18. Madison MC, Landers CT, Gu B-H, Chang C-Y, Tung H-Y, You R, Hong MJ, Baghaei N, Song L-Z, Porter P, Putluri N, Salas R, Gilbert BE, Levental I, Campen MJ, Corry DB, Kheradmand F: Electronic cigarettes disrupt lung lipid homeostasis and innate immunity independent of nicotine. J Clin Invest 2019, 129: 4290-4304

19. Espinoza-Derout J, Hasan KM, Shao XM, Jordan MC, Sims C, Lee DL, Sinha S, Simmons Z, Mtume N, Liu Y, Roos KP, SinhaHikim AP, Friedman TC: Chronic intermittent electronic cigarette exposure induces cardiac dysfunction and atherosclerosis in apolipoprotein-E knockout mice. Am J Physiol Heart Circ Physiol 2019, 317:H445-H459

20. Tang M-S, Wu X-R, Lee H-W, Xia Y, Deng F-M, Moreira AL, Chen L-C, Huang WC, Lepor H: Electronic-cigarette smoke induces lung adenocarcinoma and bladder urothelial hyperplasia in mice. Proc Natl Acad Sci U S A 2019, 116:21727-21731

21. LoPachin RM, Gavin T: Molecular mechanisms of aldehyde toxicity: a chemical perspective. Chem Res Toxicol 2014, 27:1081-1091

22. Bitzer ZT, Goel R, Reilly SM, Bhangu G, Trushin N, Foulds J, Muscat J, Richie JP: Emissions of free radicals, carbonyls, and nicotine from the NIDA standardized research electronic cigarette and comparison to similar commercial devices. Chem Res Toxicol 2019, $32: 130-138$

23. Lee M-S, LeBouf RF, Son Y-S, Koutrakis P, Christiani DC: Nicotine, aerosol particles, carbonyls and volatile organic compounds in tobacco- and menthol-flavored e-cigarettes. Environ Health 2017, $16: 42$

24. Goniewicz ML, Smith DM, Edwards KC, Blount BC, Caldwell KL, Feng J, Wang L, Christensen C, Ambrose B, Borek N, van Bemmel D, Konkel K, Erives G, Stanton CA, Lambert E, Kimmel HL, Hatsukami D, Hecht SS, Niaura RS, Travers M, Lawrence C, Hyland AJ: Comparison of nicotine and toxicant exposure in users of electronic cigarettes and combustible cigarettes. JAMA Netw Open 2018, 1:e185937

25. Cirillo S, Vivarelli F, Turrini E, Fimognari C, Burattini S, Falcieri E, Rocchi MBL, Cardenia V, Rodriguez-Estrada MT, Paolini M, Canistro D: The customizable e-cigarette resistance influences toxicological outcomes: lung degeneration, inflammation and oxidative stress-induced in a rat model. Toxicol Sci 2019, 172:132

26. Burke A, Fitzgerald GA: Oxidative stress and smoking-induced vascular injury. Prog Cardiovasc Dis 2003, 46:79-90

27. Bitzer ZT, Goel R, Reilly SM, Foulds J, Muscat J, Elias RJ, Richie JP: Effects of solvent and temperature on free radical formation in electronic cigarette aerosols. Chem Res Toxicol 2018, 31:4-12

28. Moheimani RS, Bhetraratana M, Yin F, Peters KM, Gornbein J, Araujo JA, Middlekauff HR: Increased cardiac sympathetic activity and oxidative stress in habitual electronic cigarette users: implications for cardiovascular risk. JAMA Cardiol 2017, 2:278-284

29. Dvorkin-Gheva A, Vanderstocken G, Yildirim AÖ, Brandsma C-A, Obeidat M, Bossé Y, Hassell JA, Stampfli MR: Total particulate matter concentration skews cigarette smoke's gene expression profile. ERJ Open Res 2016, 2:00029-02016

30. Rajagopalan S, Al-Kindi SG, Brook RD: Air pollution and cardiovascular disease: JACC state-of-the-art review. J Am Coll Cardiol 2018, 72:2054-2070

31. Shaffer RM, Sheppard L, Peskind ER, Zhang J, Adar SD, Li G: Fine particulate matter exposure and cerebrospinal fluid markers of vascular injury. J Alzheimers Dis 2019, 71:1015-1025

32. Manigrasso M, Buonanno G, Stabile L, Morawska L, Avino P: Particle doses in the pulmonary lobes of electronic and conventional cigarette users. Environ Pollut 2015, 202:24-31

33. Stepanov I, Sebero E, Wang R, Gao Y-T, Hecht SS, Yuan J-M: Tobacco-specific N-nitrosamine exposures and cancer risk in the 
Shanghai Cohort Study: remarkable coherence with rat tumor sites. Int J Cancer 2014, 134:2278-2283

34. Dawkins LE, Kimber CF, Doig M, Feyerabend C, Corcoran O: Self-titration by experienced e-cigarette users: blood nicotine delivery and subjective effects. Psychopharmacology (Berl) 2016, 233:2933-2941

35. Kosmider L, Jackson A, Leigh N, O'Connor R, Goniewicz ML: Circadian puffing behavior and topography among e-cigarette users. Tob Regul Sci 2018, 4:41-49

36. Schick SF, Blount BC, Jacob P, Saliba NA, Bernert JT, El Hellani A, Jatlow P, Pappas RS, Wang L, Foulds J, Ghosh A, Hecht SS, Gomez JC, Martin JR, Mesaros C, Srivastava S, St Helen G, Tarran R, Lorkiewicz PK, Blair IA, Kimmel HL, Doerschuk CM, Benowitz NL, Bhatnagar A: Biomarkers of exposure to new and emerging tobacco delivery products. Am J Physiol Lung Cell Mol Physiol 2017, 313:L425-L452

37. Freund KM, Belanger AJ, D'Agostino RB, Kannel WB: The health risks of smoking: the Framingham Study: 34 years of follow-up. Ann Epidemiol 1993, 3:417-424

38. Csordas A, Bernhard D: The biology behind the atherothrombotic effects of cigarette smoke. Nat Rev Cardiol 2013, 10:219-230

39. Bonetti PO, Lardi E, Geissmann C, Kuhn MU, Brüesch H, Reinhart WH: Effect of brief secondhand smoke exposure on endothelial function and circulating markers of inflammation. Atherosclerosis 2011, 215:218-222

40. Heiss C, Amabile N, Lee AC, Real WM, Schick SF, Lao D, Wong ML, Jahn S, Angeli FS, Minasi P, Springer ML, Hammond SK, Glantz SA, Grossman W, Balmes JR, Yeghiazarians Y: Brief secondhand smoke exposure depresses endothelial progenitor cells activity and endothelial function: sustained vascular injury and blunted nitric oxide production. J Am Coll Cardiol 2008, 51:1760-1771

41. Tőkés-Füzesi M, Ruzsics I, Rideg O, Kustán P, Kovács GL, Molnár T: Role of microparticles derived from monocytes, endothelial cells and platelets in the exacerbation of COPD. Int J Chron Obstruct Pulmon Dis 2018, 13:3749-3757

42. Kuhlmann CRW, Trümper JRFC, Tillmanns H, Alexander Schaefer C, Erdogan A: Nicotine inhibits large conductance $\mathrm{Ca}(2+)$-activated $\mathrm{K}(+)$ channels and the NO/-cGMP signaling pathway in cultured human endothelial cells. Scand Cardiovasc J 2005, 39:348-352

43. Antoniewicz L, Brynedal A, Hedman L, Lundbäck M, Bosson JA: Acute effects of electronic cigarette inhalation on the vasculature and the conducting airways. Cardiovasc Toxicol 2019, 19: $441-450$

44. Moheimani RS, Bhetraratana M, Peters KM, Yang BK, Yin F, Gornbein J, Araujo JA, Middlekauff HR: Sympathomimetic effects of acute e-cigarette use: role of nicotine and non-nicotine constituents. J Am Heart Assoc 2017, 6:e006579

45. D'Ruiz CD, O'Connell G, Graff DW, Yan XS: Measurement of cardiovascular and pulmonary function endpoints and other physiological effects following partial or complete substitution of cigarettes with electronic cigarettes in adult smokers. Regul Toxicol Pharmacol 2017, 87:36-53

46. Caporale A, Langham MC, Guo W, Johncola A, Chatterjee S, Wehrli FW: Acute effects of electronic cigarette aerosol inhalation on vascular function detected at quantitative MRI. Radiology 2019, 293: 97-106

47. Carnevale R, Sciarretta S, Violi F, Nocella C, Loffredo L, Perri L, Peruzzi M, Marullo AGM, De Falco E, Chimenti I, Valenti V, Biondi-Zoccai G, Frati G: Acute impact of tobacco vs electronic cigarette smoking on oxidative stress and vascular function. Chest 2016, 150:606-612

48. Ikonomidis I, Vlastos D, Kourea K, Kostelli G, Varoudi M, Pavlidis G, Efentakis P, Triantafyllidi H, Parissis J, Andreadou I, Iliodromitis E, Lekakis J: Electronic cigarette smoking increases arterial stiffness and oxidative stress to a lesser extent than a single conventional cigarette: an acute and chronic study. Circulation 2018, 137:303-306

49. Chatterjee S, Tao J-Q, Johncola A, Guo W, Caporale A, Langham MC, Wehrli FW: Acute exposure to e-cigarettes causes inflammation and pulmonary endothelial oxidative stress in nonsmoking, healthy young subjects. Am J Physiol Lung Cell Mol Physiol 2019, 317:L155-L166

50. Chaumont M, de Becker B, Zaher W, Culié A, Deprez G, Mélot C, Reyé F, Van Antwerpen P, Delporte C, Debbas N, Boudjeltia KZ, van de Borne P: Differential effects of e-cigarette on microvascular endothelial function, arterial stiffness and oxidative stress: a randomized crossover trial. Sci Rep 2018, 8:10378

51. Alzahrani T, Pena I, Temesgen N, Glantz SA: Association between electronic cigarette use and myocardial infarction. Am J Prev Med 2018, 55:455-461

52. Osei AD, Mirbolouk M, Orimoloye OA, Dzaye O, Uddin SMI, Benjamin EJ, Hall ME, DeFilippis AP, Stokes A, Bhatnagar A, Nasir K, Blaha MJ: Association between e-cigarette use and cardiovascular disease among never and current combustible-cigarette smokers. Am J Med 2019, 132:949-954.e2

53. Knowland D, Arac A, Sekiguchi KJ, Hsu M, Lutz SE, Perrino J, Steinberg GK, Barres BA, Nimmerjahn A, Agalliu D: Stepwise recruitment of transcellular and paracellular pathways underlies blood-brain barrier breakdown in stroke. Neuron 2014, 82: 603-617

54. Ramirez SH, Fan S, Dykstra H, Rom S, Mercer A, Reichenbach NL, Gofman L, Persidsky Y: Inhibition of glycogen synthase kinase $3 \beta$ promotes tight junction stability in brain endothelial cells by half-life extension of occludin and claudin-5. PLoS One 2013, 8:e55972

55. Abbott NJ, Patabendige AAK, Dolman DEM, Yusof SR, Begley DJ: Structure and function of the blood-brain barrier. Neurobiol Dis 2010, 37:13-25

56. Kanmogne GD, Schall K, Leibhart J, Knipe B, Gendelman HE, Persidsky Y: HIV-1 gp120 compromises blood-brain barrier integrity and enhances monocyte migration across blood-brain barrier: implication for viral neuropathogenesis. J Cereb Blood Flow Metab 2007, 27:123-134

57. Persidsky Y, Heilman D, Haorah J, Zelivyanskaya M, Persidsky R, Weber GA, Shimokawa H, Kaibuchi K, Ikezu T: Rho-mediated regulation of tight junctions during monocyte migration across the blood-brain barrier in HIV-1 encephalitis (HIVE). Blood 2006, 107: $4770-4780$

58. Lee DH, Chung MY, Lee JU, Kang DG, Paek YW: Changes of glucose transporters in the cerebral adaptation to hypoglycemia. Diabetes Res Clin Pract 2000, 47:15-23

59. Mooradian AD, Chung HC, Shah GN: GLUT-1 expression in the cerebra of patients with Alzheimer's disease. Neurobiol Aging 1997, 18:469-474

60. Persidsky Y, Ghorpade A, Rasmussen J, Limoges J, Liu XJ, Stins M, Fiala M, Way D, Kim KS, Witte MH, Weinand M, Carhart L, Gendelman HE: Microglial and astrocyte chemokines regulate monocyte migration through the blood-brain barrier in human immunodeficiency virus-1 encephalitis. Am J Pathol 1999, 155: $1599-1611$

61. Persidsky Y, Zheng J, Miller D, Gendelman HE: Mononuclear phagocytes mediate blood-brain barrier compromise and neuronal injury during HIV-1-associated dementia. J Leukoc Biol 2000, 68: 413-422

62. Hebert LE, Scherr PA, Beckett LA, Funkenstein HH, Albert MS, Chown MJ, Evans DA: Relation of smoking and alcohol consumption to incident Alzheimer's disease. Am J Epidemiol 1992, 135: 347-355

63. Ulrich J, Johannson-Locher G, Seiler WO, Stähelin HB: Does smoking protect from Alzheimer's disease? Alzheimer-type changes in 301 unselected brains from patients with known smoking history. Acta Neuropathol 1997, 94:450-454 
64. Cataldo JK, Prochaska JJ, Glantz SA: Cigarette smoking is a risk factor for Alzheimer's disease: an analysis controlling for tobacco industry affiliation. J Alzheimers Dis 2010, 19:465-480

65. Liu Q, Zhang J, Zhu H, Qin C, Chen Q, Zhao B: Dissecting the signaling pathway of nicotine-mediated neuroprotection in a mouse Alzheimer disease model. FASEB J 2007, 21:61-73

66. Jellinger KA, Attems J: Prevalence of dementia disorders in the oldest-old: an autopsy study. Acta Neuropathol 2010, 119:421-433

67. Attems J, Jellinger KA: The overlap between vascular disease and Alzheimer's disease-lessons from pathology. BMC Med 2014, 12:206

68. Cooper JK, Mungas D: Risk factor and behavioral differences between vascular and Alzheimer's dementias: the pathway to end-stage disease. J Geriatr Psychiatry Neurol 1993, 6:29-33

69. Sweeney MD, Kisler K, Montagne A, Toga AW, Zlokovic BV: The role of brain vasculature in neurodegenerative disorders. Nat Neurosci 2018, 21:1318-1331

70. Nooyens ACJ, van Gelder BM, Verschuren WMM: Smoking and cognitive decline among middle-aged men and women: the Doetinchem Cohort Study. Am J Public Health 2008, 98:2244-2250

71. Smith EE, Beaudin AE: New insights into cerebral small vessel disease and vascular cognitive impairment from MRI. Curr Opin Neurol 2018, 31:36-43

72. Gray JC, Thompson M, Bachman C, Owens MM, Murphy M, Palmer R: Associations of cigarette smoking with gray and white matter in the UK Biobank. Neuropsychopharmacology 2020, 45 . $1215-1222$

73. Anstey KJ, von Sanden C, Salim A, O'Kearney R: Smoking as a risk factor for dementia and cognitive decline: a meta-analysis of prospective studies. Am J Epidemiol 2007, 166:367-378

74. Changeux J-P: The nicotinic acetylcholine receptor: a typical "allosteric machine." Philos Trans R Soc Lond B Biol Sci 2018, 373: 20170174

75. van der Zanden EP, Hilbers FW, Verseijden C, van den Wijngaard RM, Skynner M, Lee K, Ulloa L, Boeckxstaens GE, de Jonge WJ: Nicotinic acetylcholine receptor expression and susceptibility to cholinergic immunomodulation in human monocytes of smoking individuals. Neuroimmunomodulation 2012, 19: $255-265$

76. Li X, Han X, Bao J, Liu Y, Ye A, Thakur M, Liu H: Nicotine increases eclampsia-like seizure threshold and attenuates microglial activity in rat hippocampus through the $\alpha 7$ nicotinic acetylcholine receptor. Brain Res 2016, 1642:487-496

77. Wang $\mathrm{H}$, Liao $\mathrm{H}$, Ochani $\mathrm{M}$, Justiniani $\mathrm{M}$, Lin $\mathrm{X}$, Yang L, AlAbed Y, Wang H, Metz C, Miller EJ, Tracey KJ, Ulloa L: Cholinergic agonists inhibit HMGB1 release and improve survival in experimental sepsis. Nat Med 2004, 10:1216-1221

78. Sykes AP, Brampton C, Klee S, Chander CL, Whelan C, Parsons ME: An investigation into the effect and mechanisms of action of nicotine in inflammatory bowel disease. Inflamm Res 2000 , 49:311-319

79. Chavan SS, Tracey KJ: Essential neuroscience in immunology. J Immunol 2017, 198:3389-3397

80. Gomes JP, Watad A, Shoenfeld Y: Nicotine and autoimmunity: the lotus' flower in tobacco. Pharmacol Res 2018, 128:101-109

81. Ueno H, Pradhan S, Schlessel D, Hirasawa H, Sumpio BE: Nicotine enhances human vascular endothelial cell expression of ICAM-1 and VCAM-1 via protein kinase C, p38 mitogen-activated protein kinase, NF-kappaB, and AP-1. Cardiovasc Toxicol 2006, 6: $39-50$

82. Couturier S, Bertrand D, Matter JM, Hernandez MC, Bertrand S, Millar N, Valera S, Barkas T, Ballivet M: A neuronal nicotinic acetylcholine receptor subunit (alpha 7) is developmentally regulated and forms a homo-oligomeric channel blocked by alpha-BTX. Neuron 1990, 5:847-856

83. Chatterjee PK, Al-Abed Y, Sherry B, Metz CN: Cholinergic agonists regulate JAK2/STAT3 signaling to suppress endothelial cell activation. Am J Physiol Cell Physiol 2009, 297:C1294-C1306
84. Saeed RW, Varma S, Peng-Nemeroff T, Sherry B, Balakhaneh D, Huston J, Tracey KJ, Al-Abed Y, Metz CN: Cholinergic stimulation blocks endothelial cell activation and leukocyte recruitment during inflammation. J Exp Med 2005, 201:1113-1123

85. An N, Andrukhov O, Tang Y, Falkensammer F, Bantleon H-P, Ouyang X, Rausch-Fan X: Effect of nicotine and porphyromonas gingivalis lipopolysaccharide on endothelial cells in vitro. PLoS One 2014, 9:e96942

86. Sharentuya N, Tomimatsu T, Mimura K, Tskitishvili E, KinugasaTaniguchi Y, Kanagawa T, Kimura T: Nicotine suppresses interleukin-6 production from vascular endothelial cells: a possible therapeutic role of nicotine for preeclampsia. Reprod Sci 2010, 17: $556-563$

87. Abbruscato TJ, Lopez SP, Mark KS, Hawkins BT, Davis TP: Nicotine and cotinine modulate cerebral microvascular permeability and protein expression of ZO-1 through nicotinic acetylcholine receptors expressed on brain endothelial cells. J Pharm Sci 2002, 91 : $2525-2538$

88. Hawkins BT, Abbruscato TJ, Egleton RD, Brown RC, Huber JD, Campos CR, Davis TP: Nicotine increases in vivo blood-brain barrier permeability and alters cerebral microvascular tight junction protein distribution. Brain Res 2004, 1027:48-58

89. Krafft PR, Caner B, Klebe D, Rolland WB, Tang J, Zhang JH: PHA543613 preserves blood-brain barrier integrity after intracerebral hemorrhage in mice. Stroke 2013, 44:1743-1747

90. Kimura I, Dohgu S, Takata F, Matsumoto J, Kawahara Y, Nishihira M, Sakada S, Saisho T, Yamauchi A, Kataoka Y: Activation of the $\alpha 7$ nicotinic acetylcholine receptor upregulates bloodbrain barrier function through increased claudin-5 and occludin expression in rat brain endothelial cells. Neurosci Lett 2019, 694: $9-13$

91. Rubenstein DA, Hom S, Ghebrehiwet B, Yin W: Tobacco and ecigarette products initiate Kupffer cell inflammatory responses. Mol Immunol 2015, 67:652-660

92. Kuntic M, Oelze M, Steven S, Kröller-Schön S, Stamm P, Kalinovic S, Frenis K, Vujacic-Mirski K, Bayo Jimenez MT, Kvandova M, Filippou K, Al Zuabi A, Brückl V, Hahad O, Daub S, Varveri F, Gori T, Huesmann R, Hoffmann T, Schmidt FP, Keaney JF, Daiber A, Münzel T: Short-term e-cigarette vapour exposure causes vascular oxidative stress and dysfunction: evidence for a close connection to brain damage and a key role of the phagocytic NADPH oxidase (NOX2). Eur Heart J 2019, 41:2472-2483

93. Stämpfli MR, Anderson GP: How cigarette smoke skews immune responses to promote infection, lung disease and cancer. Nat Rev Immunol 2009, 9:377-384

94. Heldt NA, Seliga A, Winfield M, Gajghate S, Reichenbach N, Yu X, Rom S, Tenneti A, May D, Gregory BD, Persidsky Y: Electronic cigarette exposure disrupts blood-brain barrier integrity and promotes neuroinflammation. Brain Behav Immun 2020, 88:363-380

95. Kaisar MA, Villalba H, Prasad S, Liles T, Sifat AE, Sajja RK, Abbruscato TJ, Cucullo L: Offsetting the impact of smoking and ecigarette vaping on the cerebrovascular system and stroke injury: is metformin a viable countermeasure? Redox Biol 2017, 13:353-362

96. Prasad S, Sajja RK, Kaisar MA, Park JH, Villalba H, Liles T, Abbruscato T, Cucullo L: Role of Nrf2 and protective effects of metformin against tobacco smoke-induced cerebrovascular toxicity. Redox Biol 2017, 12:58-69

97. Sifat AE, Vaidya B, Kaisar MA, Cucullo L, Abbruscato TJ: Nicotine and electronic cigarette (E-Cig) exposure decreases brain glucose utilization in ischemic stroke. J Neurochem 2018, 147: 204-221

98. Kaisar MA, Sivandzade F, Bhalerao A, Cucullo L: Conventional and electronic cigarettes dysregulate the expression of iron transporters and detoxifying enzymes at the brain vascular endothelium: in vivo evidence of a gender-specific cellular response to chronic cigarette smoke exposure. Neurosci Lett 2018, 682:1-9 
99. Naik P, Cucullo L: Pathobiology of tobacco smoking and neurovascular disorders: untied strings and alternative products. Fluids Barriers CNS 2015, 12:25
100. Sivandzade F, Cucullo L: Assessing the protective effect of rosiglitazone against electronic cigarette/tobacco smoke-induced blood-brain barrier impairment. BMC Neurosci 2019, 20:15 